\title{
A study of pentaquark $\Theta$ state in the chiral SU(3) quark model ${ }^{1}$
}

\author{
F. Huang, Z.Y. Zhang, Y.W. Yu \\ Institute of High Energy Physics, Beijing 100039, P.R.China \\ B.S.Zou \\ CCAST (World Laboratory), P.O. Box 8730, Beijing 100080; \\ Institute of High Energy Physics, Beijing 100039, P.R.China
}

\begin{abstract}
The structure of the pentaquark state $u u d d-\bar{s}$ is studied in the chiral $S U(3)$ quark model as well as in the extended chiral $S U(3)$ quark model, in which the vector meson exchanges are included. Four configurations of $J^{\pi}=\frac{1}{2}^{-}$and four of $J^{\pi}=\frac{1}{2}^{+}$are considered. The results show that the isospin $T=0$ state is always the lowest one for both $J^{\pi}=\frac{1}{2}^{-}$and $J^{\pi}=\frac{1}{2}^{+}$cases in various models. But the theoretical value of the lowest one is still about $200-300 \mathrm{MeV}$ higher than the experimental mass of $\Theta$. It seems that a dynamical calculation should be done for the further study.
\end{abstract}

Key words: Pentaquark state, Quark Model, Chiral Symmetry.

\section{Introduction}

Recently, LEPS Collaboration at SPring 8 [1], DIANA Collaboration at ITEP [2], CLAS Collaboration at Jefferson Lab [3] and SAPHIR Collaboration at ELSA [4] report that they observed a new resonance $\Theta$, with strangeness quantum number $S=+1$. The mass of this $\Theta$ particle is around $M_{\Theta}=1540 \mathrm{MeV}$ and the upper limit of the width is about $\Gamma_{\Theta}<25 \mathrm{MeV}$. Since it has strangeness quantum number $S=+1$, it must

\footnotetext{
${ }^{1}$ Project supported by the National Natural Science Foundation of China
} 
be a 5-quark system. The interesting problem is whether it is a strange meson-baryon molecule like state or a pentaquark state. If it is really a pentaquark state, it will be the first multi-quark state people found. There are already many theoretical works to try to explain its properties with various quark models $[5,6,7]$ or other approaches [8] . A re-analysis [9] of older experimental data on the $K^{+}$-nucleon elastic scattering process put a more stringent constraint on the width to be $\Gamma_{\Theta}<1 \mathrm{MeV}$. Since the mass of $\Theta, M_{\Theta}$, is larger than the sum of nucleon mass and kaon mass, $M_{N}+M_{K}$, it is not easy to understand why its width is so narrow, unless it has very special quantum numbers. As to the mass of $\Theta$, although it is predicted by the original chiral soliton model [10] quite well, there is no concrete calculation from quark model available yet.

In this work, we calculate the energies of the pentaquark states in chiral quark model. Four configurations of $J^{\pi}=\frac{1}{2}^{-}$and four of $J^{\pi}=\frac{1}{2}^{+}$are considered. Some qualitative information is obtained: (1) The isoscalar state, $T=0$, is always the lowest one for both cases of $J^{\pi}=\frac{1}{2}^{-}$and $J^{\pi}=\frac{1}{2}^{+}$. (2) The calculated results of the extended chiral $S U(3)$ quark model are quite similar to those of the chiral $S U(3)$ quark model, when the parameters are taken as what we used in the $N-N$ scattering calculation $[11,12]$. (3) When the size parameter is adjusted to be $0.6 \mathrm{fm}$, the energy of the lowest state $\left([4]_{o r b}[31]_{t s=01}^{\sigma f} \bar{s}, L S T=0 \frac{1}{2} 0, J^{\pi}=\frac{1}{2}^{-}\right)$, is $1670 \mathrm{MeV}$, still about $130 \mathrm{MeV}$ higher than the experimental value of the $\Theta$ mass. A dynamical calculation will be done for getting quantitative information of the $\Theta$ particle's structure.

\section{Theoretical framework}

For a $4 q-\bar{q}$ color singlet system, the $4 q$ wave function includes three parts: orbital, flavor-spin $S U(3) \times S U(2)$ and color $S U(3)$ part. In $\Theta$ particle case, its strangeness is $+1,4 q$ part only includes $\mathrm{u}$ and $\mathrm{d}$ quarks, and the anti-quark is $\bar{s}$. Four configurations for $J^{\pi}=\frac{1}{2}^{-}$are considered, they are: $\left([4]_{\text {orb }}[31]_{t s=01}^{\sigma f} \bar{s}, L S T=0 \frac{1}{2} 0, J^{\pi}=\right.$ $\left.\frac{1}{2}^{-}\right), \quad\left([4]_{o r b}[31]_{t s=10}^{\sigma f} \bar{s}, L S T=0 \frac{1}{2} 1, J^{\pi}=\frac{1}{2}^{-}\right), \quad\left([4]_{o r b}[31]_{t s=11}^{\sigma f} \bar{s}, L S T=\right.$ $\left.0 \frac{1}{2} 1, J^{\pi}=\frac{1}{2}^{-}\right)$and $\left([4]_{o r b}[31]_{t s=21}^{\sigma f} \bar{s}, L S T=0 \frac{1}{2} 2, J^{\pi}=\frac{1}{2}^{-}\right)$. We also considered 4 configurations for $J^{\pi}=\frac{1}{2}^{+}: \quad\left([31]_{\text {orb }}[4]_{t s=00}^{\sigma f} \bar{s}, L S T=1 \frac{1}{2} 0, J^{\pi}=\frac{1}{2}^{+}\right)$, $\left([31]_{\text {orb }}[4]_{t s=11}^{\sigma f} \bar{s}, L S T=1 \frac{1}{2} 1, J^{\pi}=\frac{1}{2}^{+}\right),\left([31]_{o r b}[4]_{t s=11}^{\sigma f} \bar{s}, L S T=1 \frac{3}{2} 1, J^{\pi}=\frac{1}{2}^{+}\right)$ and $\left([31]_{o r b}[4]_{t s=22}^{\sigma f} \bar{s}, L S T=1 \frac{3}{2} 2, J^{\pi}=\frac{1}{2}^{+}\right)$. The color part of them is $[211]^{c}$, 
i.e. $(\lambda \mu)_{c}=(10)$, combining (01) of $\bar{s}$, the total quantum number in color space is singlet. For $J^{\pi}=\frac{1}{2}^{-}$states, color $[211]^{c}$ with spin-flavor $[31]^{\sigma f}$ constructs the total anti-symmetric structure of the $4 q$ part, and for $J^{\pi}=\frac{1}{2}^{+}$states, [31 $]_{\text {orb }}$ replaces $[31]^{\sigma f}$ to make the anti-symmetrization.

In the chiral $S U(3)$ quark model the Hamiltonian of the system can be written as

$$
H=\sum_{i} T_{i}-T_{G}+\sum_{i<j=1-4} V_{i j}+\sum_{i=1-4} V_{i 5}
$$

where $\sum_{i} T_{i}-T_{G}$ is the kinetic energy of the system, $V_{i j}, i, j=1-4$ and $V_{i 5}, i=1-4$ represent the interactions between quark-quark $(q-q)$ and quark-anti-quark $(q-\bar{q})$ respectively.

$$
V_{i j}=V_{i j}^{c o n f}+V_{i j}^{O G E}+V_{i j}^{c h}
$$

$V_{i j}^{\text {conf }}$ is the confinement potential taken as the quadratic form,

$$
V_{i j}^{c o n f}=-a_{i j}^{c}\left(\lambda_{i}^{c} \cdot \lambda_{j}^{c}\right) r_{i j}^{2}-a_{i j}^{c 0}\left(\lambda_{i}^{c} \cdot \lambda_{j}^{c}\right),
$$

and $V_{i j}^{O G E}$ is the one gluon exchange (OGE) interaction,

$$
\begin{aligned}
V_{i j}^{O G E} & =\frac{1}{4} g_{i} g_{j}\left(\lambda_{i}^{c} \cdot \lambda_{j}^{c}\right)\left\{\frac{1}{r_{i j}}-\frac{\pi}{2} \delta\left(\vec{r}_{i j}\right)\left(\frac{1}{m_{q i}^{2}}+\frac{1}{m_{q j}^{2}}\right.\right. \\
& \left.\left.+\frac{4}{3} \frac{1}{m_{q i} m_{q j}}\left(\overrightarrow{\sigma_{i}} \cdot \overrightarrow{\sigma_{j}}\right)\right)\right\}+V_{\text {tensor }}^{O G E}+V_{\vec{\ell} \cdot \vec{s}}^{O G E}
\end{aligned}
$$

$V_{i j}^{c h}$ represents the interactions from chiral field couplings. In the chiral $S U(3)$ quark model $V_{i j}^{c h}$ includes scalar meson exchange $V_{i j}^{s}$, pseudo-scalar meson exchange $V_{i j}^{p s}$, and in the extended chiral $S U(3)$ quark model, vector meson exchange $V_{i j}^{v}$ potentials are also included,

$$
V_{i j}^{c h}=\sum_{a=0}^{8} V_{s_{a}}\left(\vec{r}_{i j}\right)+\sum_{a=0}^{8} V_{p s_{a}}\left(\vec{r}_{i j}\right)+\sum_{a=0}^{8} V_{v_{a}}\left(\vec{r}_{i j}\right) .
$$

Their expressions can be found in Refs.[11, 12]. The interaction between $q$ and $\bar{q}$ includes two parts: direct interaction and annihilation part,

$$
\begin{gathered}
V_{i 5}=V_{q \bar{q}}^{d i r}+V_{q \bar{q}}^{a n n}, \\
V_{q \bar{q}}^{d i r}=V_{q \bar{q}}^{c o n f}+V_{q \bar{q}}^{O G E}+V_{q \bar{q}}^{c h},
\end{gathered}
$$


with

$$
V_{q \bar{q}}^{c h}(\vec{r})=\sum_{i}(-1)^{G_{i}} V_{q q}^{c h, i}(\vec{r})
$$

Here $(-1)^{G_{i}}$ describes the $\mathrm{G}$ parity of the ith meson. For the $\Theta$ particle case, $q \bar{q}$ can only annihilate into $K$ and $K^{*}$ mesons, thus $V_{i 5}^{a n n}$ can be expressed as:

$$
V_{i 5}^{a n n}=V_{a n n}^{K}+V_{a n n}^{K^{*}}
$$

with

$$
\begin{aligned}
V_{a n n}^{K}= & \tilde{g}_{\text {ch }}^{2} \frac{1}{\left(\tilde{m}+\tilde{m}_{s}\right)^{2}-m_{K}^{2}}\left(\frac{1-\vec{\sigma}_{q} \cdot \vec{\sigma}_{\bar{q}}}{2}\right)_{\text {spin }}\left(\frac{2+3 \lambda_{q} \cdot \lambda_{\bar{q}}^{*}}{6}\right)_{c o l o r} \\
& \left(\frac{19}{9}+\frac{1}{6} \lambda_{q} \cdot \lambda_{\bar{q}}^{*}\right)_{\text {flavor }} \delta\left(\vec{r}_{q}-\vec{r}_{\bar{q}}\right),
\end{aligned}
$$

and

$$
\begin{aligned}
V_{a n n}^{K^{*}=} & \tilde{g}_{\text {chv }}^{2} \frac{1}{\left(\tilde{m}+\tilde{m}_{s}\right)^{2}-m_{K^{*}}^{2}}\left(\frac{3+\vec{\sigma}_{q} \cdot \vec{\sigma}_{\bar{q}}}{2}\right)_{\text {spin }}\left(\frac{2+3 \lambda_{q} \cdot \lambda_{\bar{q}}^{*}}{6}\right)_{c o l o r} \\
& \left(\frac{19}{9}+\frac{1}{6} \lambda_{q} \cdot \lambda_{\bar{q}}^{*}\right)_{\text {flavor }} \delta\left(\vec{r}_{q}-\vec{r}_{\bar{q}}\right) .
\end{aligned}
$$

Where $\tilde{g}_{c h}$ and $\tilde{g}_{c h v}$ are the coupling constants of pseudo-scalar-scalar chiral field and vector chiral field in the annihilation case respectively. $\tilde{m}$ represents the effective quark mass. Actually, $\tilde{m}$ is quark momentum dependent, here we treat it as an effective mass.

Using these two models, we did an adiabatic approximation calculation to study the energies of the $(u u d d-\bar{s})$ system.

\section{Results and discussions}

First, we carry on the calculation by taking the parameters which can reasonably reproduce the experimental data of $N-N$ and $Y-N$ scattering $[11,12]$. In the chiral $S U(3)$ quark model, besides pseudo-scalar and scalar fields coupling, $O G E$ interaction is still there to offer part of the short range repulsion, as well as in the extended chiral $S U(3)$ quark model, the $O G E$ interaction is almost replaced by the vector meson exchanges. About the annihilation interaction between $u(d)-\bar{s}$, it is a complicated problem, in Eqs. (10) and (11), the quark effective masses $\tilde{m}$ and $\tilde{m}_{s}$, as well as the annihilation coupling constants $\tilde{g}_{c h}$ and $\tilde{g}_{c h v}$ are subject to significant uncertainties. In 
our calculation, we treat $\left(\tilde{m}+\tilde{m}_{s}\right), \tilde{g}_{c h}$ and $\tilde{g}_{c h v}$ as parameters, and adjust them to fit the masses of $K$ and $K^{*}$ mesons, named case I. In case II, we omitted the annihilation interaction in the calculation to see its effects. All results of 4 configurations of $J^{\pi}=\frac{1}{2}^{-}$ and 4 of $J^{\pi}=\frac{1}{2}^{+}$are listed in Table 1 .

¿From Table 1, one can see that: (1) The isoscalar state $(T=0)$ is always the lowest state both in $J^{\pi}=\frac{1}{2}^{-}$and in $J^{\pi}=\frac{1}{2}^{+}$cases, and $\left([4]_{\text {orb }}[31]_{t s=01}^{\sigma f} \bar{s}, L S T=\right.$ $0 \frac{1}{2} 0, J^{\pi}=\frac{1}{2}^{-}$) is always the lowest one in different models. (2) The results of the chiral $S U(3)$ quark model and the extended chiral $S U(3)$ quark model are quite similar, although the short range interactions of these two models are different, one is from $O G E$ and the other is from vector meson exchanges. (3) The annihilation interactions offer attraction to the states of $J^{\pi}=\frac{1}{2}^{-}$and repulsion to $J^{\pi}=\frac{1}{2}^{+}$states. (4) When the annihilation interaction is considered, the energy of the lowest state, $\left([4]_{\text {orb }}[31]_{t s=01}^{\sigma f} \bar{s}, L S T=0 \frac{1}{2} 0, J^{\pi}=\frac{1}{2}^{-}\right)$, is about $250-300 \mathrm{MeV}$ higher than the experimental value of the $\Theta$ mass.

We tried to adjust the size parameter $b_{u}$ to be larger to see the influence. As an example, the results of $b_{u}=0.6 \mathrm{fm}$ in the chiral $S U(3)$ quark model are given in Table 2. In this case, the energies of all states become smaller, caused by the kinetic energy of the system is reduced for larger $b_{u}$. When the annihilation interaction is included (case I), the energy of the lowest state, $\left([4]_{\text {orb }}[31]_{t s=01}^{\sigma f} \bar{s}, L S T=0 \frac{1}{2} 0, J^{\pi}=\frac{1}{2}^{-}\right)$, is $1670 \mathrm{MeV}$, about $130 \mathrm{MeV}$ higher than the $\Theta^{\prime}$ mass.

In our results, the states of $J^{\pi}=\frac{1}{2}^{-}$are always lower than those of $J^{\pi}=\frac{1}{2}^{+}$, even in the extended chiral $S U(3)$ quark model, in which the $O G E$ interaction is almost totally replaced by vector meson exchanges. According to Stancu and Riska's argument [6], the state of $T=0, J^{\pi}=\frac{1}{2}^{+}$can be lower than the state of $T=0, J^{\pi}=\frac{1}{2}^{-}$, because the spin-flavor dependent interactions from Goldstone-Boson exchange potential offer more attractions to the state of $T=0, J^{\pi}=\frac{1}{2}^{+}$. In our calculation, it is true that $\pi$ and $\rho$ meson exchanges do contribute very strong attractions to the state of $T=0, J^{\pi}=\frac{1}{2}^{+}$, but when the interactions between $u(d)$ and $\bar{s}$ are included, especially the annihilation terms are considered, the state of $T=0, J^{\pi}=\frac{1}{2}^{-}$gets more attractions. This is because that among 4 pairs $u(d)-\bar{s}$ interactions, the state of $T=0, J^{\pi}=\frac{1}{2}^{-}$has 1 
Table 1: Energies of pentaquark states in different chiral quark model

\begin{tabular}{|c|c|c|c|c|}
\hline configuration & \multicolumn{2}{|c|}{$\begin{array}{c}\text { Chiral } S U(3) \text { Quark Model } \\
b_{u}=0.50 \mathrm{fm}\end{array}$} & \multicolumn{2}{|c|}{$\begin{array}{l}\text { Ex. Chiral } S U(3) \text { Quark Model } \\
\qquad b_{u}=0.45 \mathrm{fm}\end{array}$} \\
\hline$J^{\pi}=\frac{1}{2}^{-}$ & \multicolumn{2}{|c|}{$(\mathrm{MeV})$} & I & \\
\hline$[4]_{\text {orb }}[31]_{t s=01}^{\sigma f} \bar{s}$ & 1801 & 1957 & 1843 & 2091 \\
\hline$[4]_{\text {orb }}[31]_{t s=10}^{\sigma f} \bar{s}$ & 2049 & 2128 & 2089 & 2170 \\
\hline$[4]_{o r b}[31]_{t s=11}^{\sigma f} \bar{s}$ & 2117 & 2190 & 2115 & 2193 \\
\hline$[4]_{\text {orb }}[31]_{t s=21}^{\sigma f} \bar{s}$ & 2323 & 2369 & 2314 & 2334 \\
\hline$J^{\pi}=\frac{1}{2}^{+}$ & \multicolumn{2}{|c|}{$\begin{array}{l}\mathrm{I} \\
(M e V)^{\mathrm{II}}\end{array}$} & \multicolumn{2}{|c|}{ I ${ }^{\mathrm{II}}$} \\
\hline$[31]_{\text {orb }}[4]_{t s=00}^{\sigma f} \bar{s}$ & 2271 & 2185 & 2270 & 2253 \\
\hline $\begin{array}{r}{[31]_{\text {orb }}[4]_{t s=11}^{\sigma f} \bar{s}} \\
\left(S=\frac{1}{2}\right)\end{array}$ & 2308 & 2235 & 2296 & 2310 \\
\hline $\begin{array}{r}{[31]_{\text {orb }}[4]_{t s=11}^{\sigma f} \bar{s}} \\
\left(S=\frac{3}{2}\right)\end{array}$ & 2362 & 2282 & 2367 & 2337 \\
\hline$[31]_{\text {orb }}[4]_{t s=22}^{\sigma f} \bar{s}$ & 2426 & 2367 & 2412 & 2435 \\
\hline
\end{tabular}


Table 2: Energies of pentaquark states in chiral $S U(3)$ quark model with $b_{u}=0.6 \mathrm{fm}$

\begin{tabular}{|c|c|c|c|}
\hline configuration & \multicolumn{3}{|c|}{$\begin{array}{l}\text { Chiral } S U(3) \text { Quark Model } \\
\qquad\left(b_{u}=0.60 \mathrm{fm}\right)\end{array}$} \\
\hline$J^{\pi}=\frac{1}{2}^{-}$ & $\mathrm{I}$ & $\begin{array}{c}\text { II } \\
(M e V\end{array}$ & III \\
\hline$[4]_{\text {orb }}[31]_{t s=01}^{\sigma f} \bar{s}$ & 1672 & 1867 & 2027 \\
\hline$[4]_{\text {orb }}[31]_{t s=10}^{\sigma f} \bar{s}$ & 1940 & 1990 & 2039 \\
\hline$[4]_{\text {orb }}[31]_{t s=11}^{\sigma f} \bar{s}$ & 1983 & 2026 & 2051 \\
\hline$[4]_{\text {orb }}[31]_{t s=21}^{\sigma f} \bar{s}$ & 2103 & 2124 & 2090 \\
\hline$J^{\pi}=\frac{1}{2}^{+}$ & \multicolumn{3}{|c|}{$(\mathrm{MeV})$} \\
\hline$[31]_{\text {orb }}[4]_{t s=00}^{\sigma f} \bar{s}$ & 2105 & 2016 & 1997 \\
\hline $\begin{aligned} {[31]_{o r b}[4]_{t s=11}^{\sigma f} \bar{s} } \\
\left(S=\frac{1}{2}\right)\end{aligned}$ & 2124 & 2051 & 2018 \\
\hline $\begin{array}{r}{[31]_{o r b}[4]_{t s=11}^{\sigma f} \bar{s}} \\
\left(S=\frac{3}{2}\right)\end{array}$ & 2145 & 2070 & 2018 \\
\hline$[31]_{\text {orb } b}[4]_{t s=22}^{\sigma f} \bar{s}$ & 2177 & 2122 & 2051 \\
\hline
\end{tabular}

pair $u-\bar{s}$ of $(0 s)^{2}$ with spin $s=0$ and color singlet $(00)_{c}$ (i.e. $K$ meson's quantum numbers) and $\frac{1}{3}$ pair of $(0 s)^{2} s=1(00)_{c}$, the other part is color octet, but the state of $T=0, J^{\pi}=\frac{1}{2}^{+}$only has $\frac{1}{12}$ pair of $(0 s)^{2} s=0(00)_{c}, \frac{1}{4}$ pair of $(0 s 0 p) s=0(00)_{c}$, $\frac{1}{4}$ pair of $(0 s)^{2} s=1(00)_{c}, \frac{3}{4}$ pair of $(0 s 0 p) s=1(00)_{c}$ and the other part is color octet. If we take the annihilation interaction to fit the masses of $K$ and $K^{*}$, the state of $T=0, J^{\pi}=\frac{1}{2}^{-}$must be the lowest. In Table 2, case II is of the annihilation interactions omitted, and the results of without any interactions between $4 q$ and $\bar{s}$ are given in column III. One sees from Table 2 that when the interactions between $4 q$ and $\bar{s}$ are all omitted, the state of $T=0, J^{\pi}=\frac{1}{2}^{+}$can be the lowest one, but its energy $(1997 \mathrm{MeV})$ is much higher than the $\Theta$ 's mass. 


\section{Conclusions.}

The structures of pentaquark states are studied by an adiabatic approximation calculation in the chiral quark model. When the interactions between $4 q$ and $\bar{s}$ are considered, especially the parameters in the annihilation interactions are fixed by fitting the masses of $K$ and $K^{*}$, our results show that state $T=0, J^{\pi}=\frac{1}{2}^{-}$is the lowest one, and its energy is about $150-300 \mathrm{MeV}$ higher than the $\Theta$ 's mass. If we omit the interactions be-

tween $4 q$ and $\bar{s}$, then the state $T=0, J^{\pi}=\frac{1}{2}^{+}$can be lower then state $T=0, J^{\pi}=\frac{1}{2}^{-}$, in agreement with what is claimed in Ref.[6]. But the mass will be more than 400 $\mathrm{MeV}$ higher than the observed $\Theta$ mass. This means that how to treat the annihilation interaction reasonably is very important in the calculation. On the other hand, even without omitting the interactions between $4 q$ and $\bar{s}$, all of the results of various models with different parameters in this adiabatic approximation calculation still give the lowest mass for the $u u d d-\bar{s}$ pentaquark at least more than $150 \mathrm{MeV}$ above the observed mass of $\Theta$. Furthermore, its $T=0, J^{\pi}=\frac{1}{2}^{-}$configuration has a potential s-wave KN fall-apart mode [7] and hence is very difficult to explain the very narrow width of the observed width of $\Theta$. It seems that it is impossible to reproduce the observed low mass and narrow width of $\Theta$ by quark models with reasonable model parameters in the adiabatic approximation and a dynamical calculation may be necessary for the further study. 


\section{References}

[1] T. Nakano, D.S. Ahn, et. al., Phys. Rev. Lett., 91, 2003, 012002.

[2] V.V. Barmin et. al., hep-ex/0304040 .

[3] S. Stepanyan, K. Hicks, et. al., hep-ex/0307018 v3, 16 Jul 2003.

[4] J. Barth, W. Braun, et. al., hep-ex/0307083 v3, 6 Aug 2003.

[5] Simon Capstick and Philip R. Page, hep-ph/0307019 v2, 7 Aug 2003.

[6] FI. Stancu and D.O. Riska, hep-ph/0307010 v1, 1 Jul 2003;

L.Ya Glozman, hep-ph/0308232.

[7] B.K. Jennings and K. Maltman, hep-ph/0308286.

[8] R. Jaffe and F. Wilczek, hep-ph/0307341;

F.Csikor et al, hep-lat/0309090;

S.L. Zhu, hep-ph/0307345.

[9] R.A. Arndt et. al., nucl-th/0308012

[10] D. Diakonov, V. Petnov and M. Polyakov, Z. Phys., A359, 1997, 305.

[11] Z.Y. Zhang, Y.W. Yu, P.N. Shen, L.R. Dai, A.Faessler and U.Straub, Nucl. Phys., A625, 1997, 59.

[12] L.R. Dai, Z.Y. Zhang, Y.W. Yu and P. Wang, Nucl. Phys., A727, 2003, 321. 